\title{
The Arecibo Methanol Maser Galactic Plane Survey
}

\author{
Jagadheep D. Pandian ${ }^{1}$, Paul. F. Goldsmith ${ }^{2}$ \\ and Avinash A. Deshpande ${ }^{3}$ \\ ${ }^{1}$ Max Planck Institute for Radio Astronomy, Auf dem Hügel 69, 53121 Bonn, Germany \\ e-mail: jpandian@mpifr-bonn.mpg.de \\ ${ }^{2}$ Jet Propulsion Laboratory, Pasadena, CA 91109, USA \\ ${ }^{3}$ Raman Research Institute, Bangalore 560080, India
}

\begin{abstract}
The Arecibo Methanol Maser Galactic Plane Survey (AMGPS) is a blind survey for $6.7 \mathrm{GHz}$ methanol masers in a section of the Galactic plane visible from the Arecibo radio telescope. The survey for these signposts of massive star formation is complete at a flux density level of 0.27 Jy making it the most sensitive blind survey for methanol masers carried out to date, and resulted in the detection of 86 methanol masers, 48 of which are new discoveries. The properties of methanol masers discovered in the survey are consistent with their being associated with early phases of massive star formation. The data also show the tangent point of the Carina-Sagittarius spiral arm to be around a Galactic longitude of $49.6^{\circ}$.
\end{abstract}

Keywords. surveys, stars: formation, Galaxy: structure

\section{Introduction}

The $6.7 \mathrm{GHz}$ methanol maser, discovered by Menten (1991), is the strongest of Class II methanol masers. Unlike $\mathrm{OH}$ and $\mathrm{H}_{2} \mathrm{O}$ masers, the $6.7 \mathrm{GHz}$ maser has been observed exclusively towards regions containing young, massive stars. A number of recent studies give circumstantial evidence for $6.7 \mathrm{GHz}$ methanol masers being associated with early phases of massive star formation (e.g. Ellingsen 2006; Minier et al. 2005; Minier et al. 2001). This makes the $6.7 \mathrm{GHz}$ maser a powerful tool to probe Galactic structure and massive star formation which is poorly understood.

However, many questions regarding $6.7 \mathrm{GHz}$ maser emission are still unanswered. Which phases of massive star formation are associated with the maser emission? Where in relation to the massive young stellar objects (MYSOs) are the masers excited? Studies on a statistically complete sample derived from a blind survey are required to address this question. Furthermore, do faint methanol masers have different properties compared to the bright ones? Szymczak \& Kus (2000) discovered that some IRAS sources associated with relatively weak methanol masers did not satisfy Wood \& Churchwell (1989) (hereafter WC89) and Hughes \& MacLeod (1989) (hereafter HM89) color criteria for ultracompact H II regions. Here, we present the results of a sensitive blind survey of a portion of the Galactic plane that is visible to the $305 \mathrm{~m}$ Arecibo radio telescope.

\section{The Arecibo Methanol Maser Survey}

The observations were made between June 2004 and March 2006 using the C-Band High receiver at the $305 \mathrm{~m}$ Arecibo radio telescope. The telescope has an rms pointing error of $7^{\prime \prime}$ and an average full width half maximum (FWHM) beamwidth of $40^{\prime \prime}$. The backend was configured to cover an LSR velocity range of $-70 \mathrm{~km} \mathrm{~s}^{-1}$ to $+110 \mathrm{~km} \mathrm{~s}^{-1}$ 
at a velocity resolution of $0.14 \mathrm{~km} \mathrm{~s}^{-1}$ after Hanning smoothing. The target region was covered by mosaicing 100 sub-regions, each sub-region being mapped in a rectangular grid (in right ascension and declination), the separation between grid points being $15^{\prime \prime}$ in right ascension and $20^{\prime \prime}$ in declination. The integration time per grid point was typically $0.5 \mathrm{sec}$, and the resulting noise in a spectral channel was $\sim 85 \mathrm{mJy}$ after averaging the two orthogonal linear polarizations. Candidate sources were extracted from the data cubes through automated matched filter algorithms. Candidate sources were re-observed using one or two minute position switched observations at a much higher velocity resolution of $0.03 \mathrm{~km} \mathrm{~s}^{-1}$.

We detected a total of 86 sources, 48 of which are new detections. The region $35.2^{\circ} \leqslant$ $l \leqslant 53.7^{\circ},|b| \leqslant 0.41^{\circ}$ was completely sampled, while a larger area of $34.7^{\circ} \leqslant l \leqslant 54.3^{\circ}$, $|b| \leqslant 0.54^{\circ}$ was incompletely sampled. The peak flux density of the sources ranges from $0.11 \mathrm{Jy}$ to over $750 \mathrm{Jy}$. Using simulations, we found that the probability of detection of a source in our source extraction algorithm was greater than $95 \%$ for signals that have signal to noise ratio higher than 3.0. Since the noise in the data cubes is no greater than $0.09 \mathrm{Jy}$, we conclude that our source catalog is complete at the level of $0.27 \mathrm{Jy}$. The source catalog is available in Pandian et al. (2007).

\section{Distribution in the Galaxy}

To consider the distribution of methanol masers in the Galaxy, we exclude sources that lie outside the region that is fully sampled by the survey, along with sources that have peak flux densities below our completeness limit of $0.27 \mathrm{Jy}$. This gives a sample of 72 methanol masers. Figure 1 shows the distribution of these methanol masers as a function of Galactic latitude. A Gaussian fit to the distribution has a mean at a latitude of $-0.09^{\circ}$ and a FWHM of $0.49^{\circ}$. The width of the distribution is comparable to that of embedded massive stars determined from the IRAS point source catalog (Wood \& Churchwell 1989). The fit parameters to the distribution are very similar to those obtained by Pestalozzi et al. (2005) for the general catalog of methanol masers which contains over 500 sources. Over half of the sources in the general catalog were detected in targeted surveys towards tracers of massive star formation such as ultracompact HII regions and $\mathrm{OH}$ masers. The close agreement in the distribution of AMGPS sources with that of Pestalozzi et al. (2005) gives further evidence for the association of $6.7 \mathrm{GHz}$ methanol masers with massive star formation.

\section{Implications for Galactic Structure}

Since massive stars form preferentially along spiral density waves which compress molecular gas to high densities, $6.7 \mathrm{GHz}$ methanol masers are potential probes of the spiral structure of the Galaxy. As distances to masers in the AMGPS catalog are not known precisely, and most kinematic distances suffer from the distance ambiguity, we use an $l-v$ diagram to see what information methanol masers provide regarding the structure of our Galaxy. Two constructs of the $l-v$ diagram are shown in Figure 2. The observed radial velocities are assumed to have deviations from the values observed from pure circular rotation by up to $\pm 5 \mathrm{~km} \mathrm{~s}^{-1}$. The left and right panels of Figure 2 show the loci of various spiral arms in the models of Vallee (1995) and the NE2001 model of Cordes \& Lazio (2002) respectively. The spiral arm loci are calculated using the rotation curve of Clemens (1985).

The clumping of sources around $l \sim 49.6^{\circ}$ seems to arise from the tangent point of the Carina-Sagittarius spiral arm. The model of Vallee (1995) fits this feature reasonably 


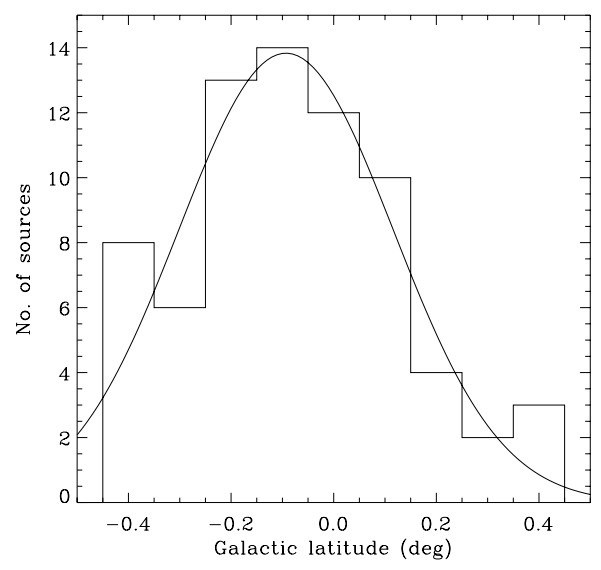

Figure 1. Distribution of methanol masers as a function of Galactic latitude. A Gaussian fit to the distribution is overlaid. The Gaussian fit has a mean at $-0.09^{\circ}$ and a FWHM of $0.49^{\circ}$.
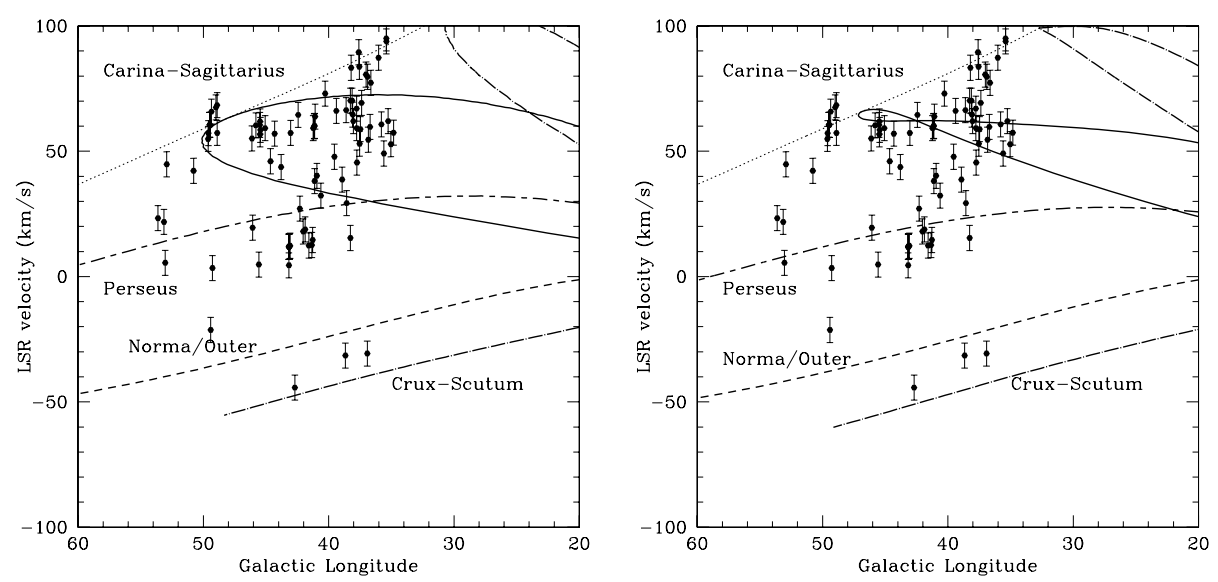

Figure 2. The methanol maser sample of AMGPS on an $l-v$ diagram. The left and right panels show the loci of spiral arms from the model of Vallee (1995) and the NE2001 model of Cordes \& Lazio (2002) respectively. The dotted line shows the tangent point velocity.

well, while the tangent point in the model of Cordes \& Lazio (2002) occurs at approximately $1^{\circ}$ lower Galactic longitude which does not fit our data. Figure 2 also shows that it is quite challenging to extract information on Galactic spiral structure from $6.7 \mathrm{GHz}$ methanol masers, since a significant fraction of sources do not lie near spiral arm loci.

\section{The number of $6.7 \mathrm{GHz}$ methanol maser sources in the Galaxy}

The number of $6.7 \mathrm{GHz}$ methanol masers in the Galaxy has been estimated by van der Walt (2005). The procedure followed is to model the distribution of young massive star forming regions as a function of Galactic longitude using a star formation law, initial mass function, and a spiral arm model of the Galaxy. This distribution is then scaled such that the number of maser sources in a chosen longitude bin coincides with the statistics from an existing survey. Since all surveys are flux limited, a minimum number of methanol masers can be derived by summing over all longitude bins. Thus, the minimum number 
of methanol masers was determined by van der Walt (2005) to be 845 , by scaling the model distribution to the observations between Galactic longitudes of $330^{\circ}$ and $340^{\circ}$.

Since our survey is an order of magnitude more sensitive more than that of existing surveys, our observations can be used to revise the minimum number of methanol masers in the Galaxy. The AMGPS detected 44 methanol masers brighter than 0.27 Jy between longitudes of $40^{\circ}$ and $50^{\circ}$. Using this number as the normalization for the longitude distribution of van der Walt (2005), the number of methanol masers in the Galaxy, brighter than $0.27 \mathrm{Jy}$, is at least 1125 . This number is a lower limit as the region $|b|>0.41^{\circ}$ is incompletely sampled by AMGPS.

Furthermore, van der Walt (2005) estimated the completeness of a survey as a function of flux density through Monte Carlo simulations. This was then used to derive the total number of methanol maser sources in the Galaxy as $1200 \pm 84$. Applying the same procedure to statistics from our survey results in the total number of maser sources to be 1197-1264, which is consistent with the analysis of van der Walt (2005).

\section{Counterparts in IRAS, MSX and NVSS catalogs}

As mentioned earlier, the rms pointing error of the telescope is $7^{\prime \prime}$, and the statistical distribution of this error is a Gaussian to good approximation. The error circle associated with any methanol maser, defined at the $95 \%$ confidence-level, is then $\sim 18^{\prime \prime}$. We thus search for counterparts within $23^{\prime \prime}$ of the methanol maser $\left(5^{\prime \prime}\right.$ being taken as the limit for association). We exclude sources in W49 and W51 from this analysis since the density of methanol masers in these regions is high enough that a given IRAS, MSX or NVSS source will lie within the search radius of more than one maser.

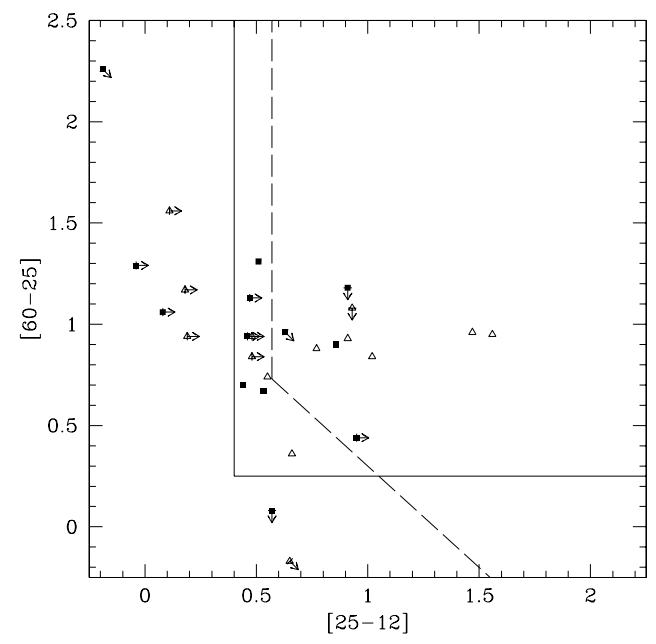

Figure 3. The color-color diagram for possible IRAS counterparts of $6.7 \mathrm{GHz}$ methanol masers discovered in AMGPS. The $\mathrm{x}$ - and y-axes show the [25-12] and [60-25] colors defined as $\log _{10}\left(F_{25} / F_{12}\right)$ and $\log _{10}\left(F_{60} / F_{25}\right)$ respectively. The colors for previous detections are shown in open triangles, while those of new detections are shown in filled squares. Color limits pointing at $45^{\circ}$ indicate upper limits in both [25-12] color and [60-25] colors. Sources to the right of the dashed lines satisfy WC89 colors for ultracompact HII regions, while sources to the right of the bold lines satisfy HM89 criteria for the same.

Carrying out this analysis, only 26 out of 76 sources have possible IRAS counterparts. This is more dramatic than results from the previous blind surveys of Ellingsen et al. 
(1996) and Szymczak et al. (2002), who found IRAS-based surveys would miss about half of the methanol maser population. The color-color diagram of these IRAS sources is shown in Figure 3. The WC89 and HM89 criteria for embedded massive stars are shown in the figure. Only six sources satisfy WC89 criteria, while a larger number of sources satisfy HM89 criteria. This shows that targeted searches for methanol masers towards IRAS color selected sources will miss a significant population of masers in the Galaxy. One of the reasons for the poor correspondence between methanol masers and IRAS sources could be source confusion in crowded fields of the Galactic plane, which resulted in many sources being not included in the point source catalog. It is to be noted that Figure 3 does not show any segregation between the previous and new detections (new detections are typically weaker); this is contrary to the result of Szymczak \& Kus (2000) who found that IRAS sources associated with weak methanol masers populated the upper left part of Fig. 3.

Only 41 out of 76 methanol masers have possible MSX point source counterparts. Visually inspecting MSX image fields, four sources are clearly associated with MSX dark clouds, while the association with dark clouds is more uncertain for an additional four sources. The lack of point source counterparts for many sources could be in part a sensitivity effect; many masers lie close to bright mid-infrared sources. Seven out of 76 sources have NVSS point sources within $23^{\prime \prime}$. This is most probably due to any HII regions associated with the methanol masers being too compact and optically thick to be detectable at $21 \mathrm{~cm}$. Thus, the lack of NVSS counterparts to most of the methanol masers suggests that they are primarily associated with phases of massive star formation prior to an ultracompact HII region. A few methanol masers do not have any tracer of star formation detected to date within $1^{\prime}$; the nature of these intriguing sources is not known at present.

\section{Conclusions}

The AMGPS has detected 48 new $6.7 \mathrm{GHz}$ methanol masers between Galactic longitudes of $34.8^{\circ}$ and $54^{\circ}$. The properties of the masers in the AMGPS catalog are consistent with them being associated with early phases of massive star formation. The total number of methanol maser sources in the Galaxy is estimated to be about 1200 .

\section{References}

Clemens, D. P. 1985, ApJ 295, 422

Cordes, J. M., \& Lazio, T. J. W. 2002, astro-ph/0207156

Ellingsen, S. P. 2006, ApJ 638, 241

Ellingsen, S. P., von Bibra, M. L., McCulloch, P. M., Norris, R. P., Deshpande, A. A., \& Phillips, C. J. 1996, MNRAS 280, 378

Hughes, V. A., \& MacLeod, G. C. 1989, AJ 97, 786

Menten, K. M. 1991, ApJ 380, L75

Minier, V., Burton, M. G., Hill, T., Pestalozzi, M. R., Purcell, C. R., Garay, G., Walsh, A. J., \& Longmore, S. 2005, A 8 A 429, 945

Minier, V., Conway, J. E., \& Booth, R. S. 2001, A\& $A$ 369, 278

Pandian, J. D., Goldsmith, P. F., \& Deshpande, A. A. 2007, ApJ 656, 255

Pestalozzi, M. R., Minier, V., \& Booth, R. S. 2005, A\&A 432, 737

Szymczak, M., \& Kus, A. J. 2000, A\&A 360, 311

Szymczak, M., Kus, A. J., Hrynek, G., Krȩpa, A., \& Pazderski, E. 2002, A\&\&A 392, 277

Vallee, J. P. 1985, ApJ 454, 119

van der Walt, J. 2005, MNRAS 360, 153

Wood, D. O. S., \& Churchwell, E. 1989, ApJ 340, 265 